\title{
Effects of Manganese Hydroxychloride on Growth Performance, Antioxidant Capacity, Tibia Parameters and Manganese Deposition of Broilers
}

\author{
Yongbo Sun, Shixia Geng, Tianyao Yuan, Ying Liu, Yuxin Zhang, Yuting Di, Juntao Li and Liying Zhang * \\ State Key Laboratory of Animal Nutrition, Ministry of Agriculture and Rural Affairs Feed Industry Centre, \\ China Agricultural University, Beijing 100193, China; ybsun2014@163.com (Y.S.); gengshixia@163.com (S.G.); \\ tyyuan2018@163.com (T.Y.); liuyingcau2021@163.com (Y.L.); s20203040632@cau.edu.cn (Y.Z.); \\ dyt971004@163.com (Y.D.); wacau@163.com (J.L.) \\ * Correspondence: zhangliying@cau.edu.cn; Tel.: +86-186-1164-4832
}

check for updates

Citation: Sun, Y.; Geng, S.; Yuan, T.; Liu, Y.; Zhang, Y.; Di, Y.; Li, J.; Zhang, L. Effects of Manganese

Hydroxychloride on Growth

Performance, Antioxidant Capacity, Tibia Parameters and Manganese Deposition of Broilers. Animals 2021, 11, 3470. https://doi.org/10.3390/ ani11123470

Academic Editor:

Sylwester Świątkiewicz

Received: 9 November 2021

Accepted: 2 December 2021

Published: 6 December 2021

Publisher's Note: MDPI stays neutral with regard to jurisdictional claims in published maps and institutional affiliations.

Copyright: (c) 2021 by the authors. Licensee MDPI, Basel, Switzerland. This article is an open access article distributed under the terms and conditions of the Creative Commons Attribution (CC BY) license (https:// creativecommons.org/licenses/by/ $4.0 /)$.
Simple Summary: Manganese is a vital trace element for the growth of broilers. In order to meet the requirement of manganese in broiler production, the additives of manganese sources are usually added into the diet for broilers. Manganese hydroxychloride is a category of hydroxy trace minerals. The present study investigated the effect of dietary supplemental manganese as manganese hydroxychloride for growth performance, antioxidant capacity, tibial quality, and manganese deposition of broilers and recommended that optimal supplementation with manganese as manganese hydroxychloride in diets for broilers was $50-90 \mathrm{mg} / \mathrm{kg}$. This study provides a rational recommendation for the application of manganese hydroxychloride in broiler diets.

Abstract: This study was conducted to investigate the effects of dietary supplementation with manganese hydroxychloride (MHC) on production performance, antioxidant capacity, tibial quality, and manganese (Mn) deposition of broilers. A total of 756 one-day-old male Arbor Acres broilers were randomly allotted to 7 treatments of 6 replicates with 18 broilers per replicate. Broilers were fed corn-soybean meal basal diets supplemented of $100 \mathrm{mg} / \mathrm{kg} \mathrm{Mn}$ as Mn sulfate $\left(\mathrm{MnSO}_{4}\right)$, or 0, 20, 40, $60,80,100 \mathrm{mg} / \mathrm{kg} \mathrm{Mn}$ as MHC for 42 days. The growth performance of broilers was not affected by dietary $\mathrm{MnSO}_{4}$ or $\mathrm{MHC}(p>0.05)$, whereas the dressing percentage increased linearly $(p<0.05)$ with increasing of dietary MHC addition level. The activities of catalase (CAT) and manganese superoxide dismutase (MnSOD), and total antioxidant capability (T-AOC) in serum and liver on day 42 increased linearly $(p<0.05)$ with increasing of dietary MHC level, while malondialdehyde (MDA) concentration reduced linearly $(p<0.05)$. The length, strength, and density index of tibia increased linearly $(p<0.05)$ on day 21 as MHC supplementation level increased; there were no differences between $\mathrm{MnSO}_{4}$ group and 40-100 mg/ $\mathrm{kg}$ Mn as MHC groups in tibial parameters of broilers $(p>0.05)$. As supplemental MHC levels increased, the Mn contents in heart, liver, kidney, and tibia increased linearly on day $42(p<0.05)$. In summary, dietary supplementation with MHC improved antioxidant capacity, bone quality, and Mn contents in broilers, but no effects on growth performance were detected. Based on the results of this study, dietary inclusion of $50-90 \mathrm{mg} / \mathrm{kg} \mathrm{Mn}$ in the form of MHC to broilers is recommended.

Keywords: Mn hydroxychloride; broiler; antioxidant capacity; tibia parameters; Mn deposition

\section{Introduction}

As an essential trace element, manganese (Mn) is a component or activator of many essential enzymes, such as arginase, pyruvate carboxylase, hydrolases, etc., which are involved in carbohydrate, lipid, and protein metabolism and many crucial biochemical reactions [1,2]. It is also an essential cofactor of chondroitin sulfate synthesis that is closely related to the bone formation of broilers [3]. Otherwise, Mn plays a vital role in antioxidant 
and immune systems of animals [4-6]. As recommended by the National Research Council (NRC), the content of Mn in the diet for broilers should be at least $60 \mathrm{mg} / \mathrm{kg}$ [7]. However, the content of $\mathrm{Mn}$ is just around $30 \mathrm{mg} / \mathrm{kg}$ in the diet mainly consisting of corn and soybean meal and its utilization rate of $\mathrm{Mn}$ is very low [8]. In order to meet the requirement of $\mathrm{Mn}$ in broiler production, the additives of $\mathrm{Mn}$ sources are usually added into the diet for broilers.

At present, additives of Mn source which is usually used in broiler diets include inorganic Mn (such as Mn sulfate, Mn carbonate, and Mn oxide) and organic Mn (such as amino acid-chelated $\mathrm{Mn}$ and protein $\mathrm{Mn}$ ). For traditional inorganic sources of $\mathrm{Mn}$, they are easy to deliquescence and the utilization rate is relatively low, although they are cheaper $[9,10]$. Organic sources of Mn have shown excellent chemical stability and high absorption efficiency, but they have not been widely applied in production because of their uneven product quality, inconsistent effect, and high cost $[9,11,12]$. Thus, it is very important to develop new sources of Mn with better efficacy and relatively low cost.

Manganese hydroxychloride (MHC), also known as basic Mn chloride or tribasic Mn chloride, is a category of hydroxy trace minerals. The solubility of MHC in water is minimal, but it becomes more soluble under acidic conditions in intestine [13]. MHC contains strong covalent bonds that are similar to that of organic minerals and have a special crystalline structure, which may be beneficial for the stability in the diet and better absorption in the intestine. MHC has been approved as a feed additive by the European Food Safety Authority (EFSA) [14]. Previous studies showed that dietary supplementation with MHC could increase egg yolk and shell Mn levels of laying hens, and improve feed conversion of broilers $[15,16]$. A study conducted with pigs also showed that dietary inclusion of MHC improved the growth rate and feed intake compared with that of $\mathrm{MnSO}_{4}$ [17]. However, there is limited literature about the suitable addition level of MHC for broilers, and the relative effect compared with typical inorganic Mn has not been reported. Therefore, the main aim of this study was to explore the suitable level of Mn as MHC in the broiler diet by investigating the effects of dietary MHC supplementation on growth performance, slaughter traits, antioxidant capacity, tibial quality, and tissue Mn level of broilers.

\section{Materials and Methods}

\subsection{Experimental Material}

Manganese hydroxychloride (MHC) was supplied by Changsha Xingjia Bio-Engineering Co., Ltd. (Changsha, China). The molecular formula of $\mathrm{MHC}$ is $\mathrm{Mn}_{2}(\mathrm{OH})_{3} \mathrm{Cl}$ and the analyzed content of $\mathrm{Mn}$ was $55.64 \%$. The feed grade $\mathrm{MnSO}_{4}$ was purchased from Beijing Tongli Xingke Agricultural Technology Co., Ltd. (Beijing, China), the analyzed Mn concentration was $31.94 \%$.

\subsection{Animals, Experimental Design and Management}

This study was performed on the Fengning Research Unit of China Agricultural University (Chengde, Hebei, China). A total of 756 one-day-old Arbor Acres male broiler chicks with an average body weight of $44.28 \pm 1.74 \mathrm{~g}$ were obtained from Arbor Acres Poultry Breeding Company (Beijing, China). All birds were weighed and randomly assigned to 7 treatments with 6 replicates and 18 chicks in each replicate. The diets consisted of a corn and soybean diet with 0 (control group), 20,40,60, 80, or $100 \mathrm{mg} / \mathrm{kg} \mathrm{Mn}$ as $\mathrm{MHC}$, and $100 \mathrm{mg} / \mathrm{kg} \mathrm{Mn}$ as $\mathrm{MnSO}_{4}$ (positive control), respectively. The recommended Mn content for broilers is $120 \mathrm{mg} / \mathrm{kg}$ during 1-3 weeks and $100 \mathrm{mg} / \mathrm{kg}$ during 4-6 weeks by the Feeding Standard of Chicken in China. Additionally, we searched recent related articles on broiler experiments in Animals and also found that the amount of added $\mathrm{Mn}$ in the diet was around $100 \mathrm{mg} / \mathrm{kg}$ [18-22]. So we added $100 \mathrm{mg} / \mathrm{kg} \mathrm{Mn}$ in the form of $\mathrm{MnSO}_{4}$ as the positive control. This experiment lasted for $42 \mathrm{~d}$, and all broilers were fed with starter diets from day 0 to day 21, and then fed with grower diets until the end of the trial. All diets were fed in mash form. Except for Mn, the corn-soybean meal basal diet was formulated according to the recommendations of the nutritional requirements of 
broilers (NRC, 1994) [7]. The ingredients compositions and nutritional levels of basal diets are shown in Table 1.

Table 1. Ingredient composition and nutrient levels of the basal diets (\%, as-fed basis).

\begin{tabular}{ccc}
\hline Item & $\begin{array}{c}\text { Starter Diets } \\
\text { (0 21 Days of Age) }\end{array}$ & $\begin{array}{c}\text { Grower Diets } \\
\text { (22 42 Days of Age) }\end{array}$ \\
\hline Ingredient & & \\
Corn & 60.13 & 61.53 \\
Soybean meal & 32.50 & 31.70 \\
Fish meal & 2.00 & 0.00 \\
Soybean oil & 1.50 & 3.00 \\
Dicalcium phosphate & 1.50 & 1.70 \\
Limestone & 1.34 & 1.15 \\
DL-Methionine (98\%) & 0.23 & 0.12 \\
NaCl & 0.30 & 0.30 \\
Premix ${ }^{1}$ & 0.50 & 0.50 \\
Total & 100.00 & 100.00 \\
Nutrient composition ${ }^{2}$ & & \\
Metabolizable energy (MJ/kg) & 12.59 & 13.22 \\
Crude protein & 21.75 & 20.27 \\
Calcium & 0.98 & 0.88 \\
Total phosphorus & 0.68 & 0.63 \\
Total lysine & 1.10 & 0.95 \\
Total methionine + cysteine & 0.85 & 0.73 \\
Mn (mg/kg) & 34.4 & 37.0 \\
\hline
\end{tabular}

${ }^{1}$ Premix provided per $\mathrm{kg}$ of diet: vitamin A, $9000 \mathrm{IU}$; vitamin D3, $3000 \mathrm{IU}$; vitamin E, $24 \mathrm{mg}$; vitamin K3, $1.8 \mathrm{mg}$; vitamin B1, $2.0 \mathrm{mg}$; riboflavin, $5 \mathrm{mg}$; vitamin B6, $3.0 \mathrm{mg}$; vitamin B12, $0.1 \mathrm{mg}$; nicotinic acid, $40 \mathrm{mg}$; calcium pantothenate, $15 \mathrm{mg}$; folic acid, $1 \mathrm{mg}$; biotin, $0.05 \mathrm{mg}$; choline chloride, $500 \mathrm{mg}$; Fe, $80 \mathrm{mg}$; $\mathrm{Cu}, 20 \mathrm{mg}$; Zn, $90 \mathrm{mg}$; iodine, $0.35 \mathrm{mg}$; Se, $0.3 \mathrm{mg} .{ }^{2}$ Metabolizable energy was calculated values using NRC (1994) values; others were analyzed values.

The experiment was performed on the Fengning Research Base of China Agricultural University (Chengde, Hebei, China). All broilers were raised in 3-layer cages $\left(0.093 \mathrm{~m}^{2}\right.$ per bird) with six birds per cage in an environmentally controlled room. Feed and water were offered ad libitum throughout the experiment. The lighting program was $23 \mathrm{~h}$ light: $1 \mathrm{~h}$ dark per day. The room temperature was maintained at $35^{\circ} \mathrm{C}$ for the first 3 day and gradually reduced by $3{ }^{\circ} \mathrm{C}$ each week until it reached to final temperature of $24^{\circ} \mathrm{C}$. All chicks were inoculated with Newcastle disease vaccine on day 7 and day 21 , and infectious bursal disease vaccine on day 14 and day 28.

\subsection{Growth Performance}

Body weight and feed intake per replicate were recorded on days 21 and 42 of the experiment. Average daily gain (ADG), average daily feed intake (ADFI), and feed to gain ratio (F:G) were calculated from days 0 to 21,22 to 42 , and 0 to 42 .

\subsection{Sample Collection}

On day 21 and 42 after fasting for 12 h, 6 broilers approximating the average weight from each treatment (one bird per replicate) were selected to collect blood samples from the wing vein of broilers. Blood samples were allowed to stand for $30 \mathrm{~min}$ at room temperature, followed by centrifugation at $3600 \times g$ for $15 \mathrm{~min}$. Then, serum samples were collected and stored at $-20{ }^{\circ} \mathrm{C}$ for further analysis. The selected broilers were euthanized by jugular vein bleeding after stunning using $60 \%$ concentration of $\mathrm{CO}_{2}$ gas. The tibia, liver, heart, kidney, and breast muscle samples were collected and stored at $-20{ }^{\circ} \mathrm{C}$ for subsequent analysis. In addition, live weight, carcass weight, eviscerated weight, breast weight, thigh muscle weight, and abdominal fat weight of broilers were measured on day 42. Dressing percentage and eviscerated percentage were expressed as a percentage of its initial live weight, while breast meat percentage, leg meat percentage, and abdominal fat percentage were expressed as a percentage of the eviscerated weight. 


\subsection{Sample Analysis}

\subsubsection{Nutrients Level of Diets}

Diets were analyzed according to the methods of the Association of Official Analytical Chemists (AOAC, 2000) for total phosphorus (method 995.11), calcium (method 927.02), crude protein (method 988.05), and amino acids (method 994.12). For dietary methionine determination, performic acid oxidation was performed prior to acid hydrolysis with $6 \mathrm{M}$ $\mathrm{HCl}$. The Mn concentration of $\mathrm{MHC}, \mathrm{MnSO}_{4}$, and diets was determined by Z-2000 flame atomic absorption spectrometry (Hitachi, Tokyo, Japan).

\subsubsection{Antioxidant Capacity}

Total antioxidant capacity (T-AOC), Mn containing superoxide dismutase (MnSOD) and catalase (CAT) activities, and malondialdehyde (MDA) contents in serum and liver of broilers were measured with commercial kits (Sino-UK Institute of Biological Technology, Beijing, China) according to the manufacturer's protocol.

\subsubsection{Tibia Indicator}

After taking the left tibia, muscles, cartilage, and membranes of it were removed. The length and diameter of tibia were measured using a vernier caliper and then weighed. The density index of the tibia was measured using a dual-energy X-ray absorptiometry bone densitometer (Hologic, Bedford, MA, USA), and the breaking strength was measured using a TA.XT plus texture analyser (Stable Microsystems, Surrey, UK).

\subsubsection{Manganese Contents}

The contents of $\mathrm{Mn}$ in heart, liver, kidney, tibia, and breast muscles samples of broilers on day 42 were determined by inductively coupled plasma mass spectrometry (Agilent 7500, Agilent Technologies, Tokyo, Japan) after microwave digestions with nitric acid.

\subsection{Statistical Analysis}

All data were subjected to one-way ANOVA using the general linear model (GLM) procedure of SAS 9.2 (SAS Institute Inc., Cary, NC, USA). Differences among treatments were further compared using Duncan's multiple range test. Orthogonal polynomial contrasts were used to analyze the linear and quadratic responses to MHC levels. Also, a quadratic regression fitting curve model $\left[y=\mathrm{a} x^{2}+\mathrm{b} x+\mathrm{c}\right.$, the best addition level $\left.x=-\mathrm{b} /(2 \mathrm{a})\right]$ was performed using GraphPad Prism 7 (GraphPad Software Inc.; San Diego, CA, USA) to evaluate the optimal MHC addition levels. A $p$-value of less than 0.05 was considered to be statistically significant.

\section{Results}

\subsection{Mn Contents in Experimental Diets}

The analyzed Mn content in the starter and grower diets are presented in Table 2. The analyzed Mn concentrations in diets supplemented with $0,20,40,60,80$, or $100 \mathrm{mg} / \mathrm{kg}$ $\mathrm{Mn}$ as $\mathrm{MHC}$, and $100 \mathrm{mg} / \mathrm{kg} \mathrm{Mn}$ as $\mathrm{MnSO}_{4}$ were 34.4, 53.3, 73.1, 91.7, 110.5, 139.9, and $150.4 \mathrm{mg} / \mathrm{kg}$ for the starter diets, and 37.0, 60.8, 77.1, 99.6, 119.0, 142.5 and $151.1 \mathrm{mg} / \mathrm{kg}$ for the grower diets, respectively. 
Table 2. Analyzed manganese (Mn) contents in experimental diets.

\begin{tabular}{cccc}
\hline \multirow{2}{*}{ Item } & \multicolumn{2}{c}{ Added $\mathbf{M n}, \mathbf{~ m g} / \mathbf{k g}$} & \multicolumn{2}{c}{ Analyzed Mn Contents, $\mathbf{~ m g / k g}$} \\
\cline { 2 - 4 } & & Day 0 to 21 & Day 22 to 42 \\
\hline Mn as $\mathrm{MnSO}_{4}$ & 100 & 150.4 & 151.1 \\
\hline & 0 & 34.4 & 37.0 \\
\cline { 2 - 4 } $\begin{array}{c}\text { Mn as manganese } \\
\text { hydroxychloride }\end{array}$ & 20 & 53.3 & 60.8 \\
\cline { 2 - 4 } & 40 & 73.1 & 77.1 \\
\cline { 2 - 4 } & 60 & 91.7 & 99.6 \\
\cline { 2 - 4 } & 80 & 110.5 & 119.0 \\
\hline
\end{tabular}

\subsection{Growth Performance}

The effects of dietary supplementation with MHC on the growth performance of broilers are presented in Table 3. There were no significant differences in ADG, ADFI, and F:G among all treatments were detected during days $0-21,22-42$, and $0-42(p>0.05)$.

Table 3. Effect of dietary supplementation with manganese hydroxychloride on growth performance of broilers ${ }^{1}$.

\begin{tabular}{|c|c|c|c|c|c|c|c|c|c|c|c|}
\hline \multirow{2}{*}{ Item $^{2}$} & \multirow{2}{*}{$\begin{array}{c}\begin{array}{c}\mathrm{Mn} \text { as } \\
\mathrm{MnSO}_{4} \\
(\mathrm{mg} / \mathrm{kg})\end{array} \\
100\end{array}$} & \multicolumn{6}{|c|}{ Mn as Manganese Hydroxychloride (mg/kg) } & \multirow{2}{*}{ SEM } & \multicolumn{3}{|c|}{$p$-Value } \\
\hline & & 0 & 20 & 40 & 60 & 80 & 100 & & ANOVA & Linear & Quadratic \\
\hline \multicolumn{12}{|l|}{ Day 0 21 } \\
\hline ADG $(g)$ & 32.60 & 31.96 & 33.01 & 32.37 & 33.37 & 32.05 & 33.25 & 0.478 & 0.241 & 0.235 & 0.638 \\
\hline ADFI $(g)$ & 43.97 & 43.27 & 44.18 & 43.66 & 44.52 & 43.42 & 44.33 & 0.575 & 0.674 & 0.396 & 0.644 \\
\hline $\mathrm{F}: \mathrm{G}$ & 1.35 & 1.36 & 1.34 & 1.35 & 1.34 & 1.35 & 1.33 & 0.011 & 0.650 & 0.374 & 0.857 \\
\hline \multicolumn{12}{|l|}{ Day 22 42 } \\
\hline ADG $(\mathrm{g})$ & 71.58 & 68.86 & 69.83 & 69.46 & 72.01 & 71.71 & 70.48 & 1.357 & 0.559 & 0.145 & 0.373 \\
\hline ADFI (g) & 136.48 & 134.34 & 135.92 & 134.45 & 138.72 & 136.03 & 135.71 & 1.596 & 0.549 & 0.380 & 0.314 \\
\hline$F: G$ & 1.91 & 1.95 & 1.95 & 1.94 & 1.93 & 1.90 & 1.93 & 0.028 & 0.800 & 0.245 & 0.748 \\
\hline \multicolumn{12}{|l|}{ Day 0 42 } \\
\hline $\mathrm{ADG}(\mathrm{g})$ & 52.09 & 50.41 & 51.42 & 50.91 & 52.69 & 51.88 & 51.86 & 0.835 & 0.552 & 0.128 & 0.389 \\
\hline ADFI (g) & 90.23 & 88.81 & 90.05 & 89.06 & 91.62 & 89.73 & 90.02 & 0.941 & 0.475 & 0.299 & 0.301 \\
\hline$F: G$ & 1.73 & 1.76 & 1.75 & 1.75 & 1.74 & 1.73 & 1.74 & 0.019 & 0.891 & 0.227 & 0.780 \\
\hline
\end{tabular}

${ }^{1}$ Values are expressed as means of six replicates of 18 birds per pen. ${ }^{2}$ ADG, average daily gain; ADFI, average daily feed intake; F:G, feed: gain ratio.

\subsection{Carcass Characteristics}

As shown in Table 4 , the dressing percentage of broilers increased linearly $(p<0.05)$ on day 42 with the increase of dietary MHC addition level. The percentage of breast muscle, leg muscle, and abdominal fat had not been significantly affected by dietary MHC or $\mathrm{MnSO}_{4}(p>0.05)$.

\subsection{Antioxidant Capacity}

The effects of dietary supplementation with MHC on the antioxidant capacity in serum of broiler are shown in Table 5 . Dietary supplementation with MHC linearly increased the activities of CAT and MnSOD in serum on day $21(p<0.01)$, and the activities of serum CAT and MnSOD and T-AOC also increased (linear, $p<0.05$; quadratic, $p<0.05$ ) as supplemental MHC level increased on day $42(p<0.05)$, whereas MDA level in serum decreased linearly on day 21 and $42(p<0.01)$. No differences were observed in these parameters among $\mathrm{MnSO}_{4}$ and $40-60 \mathrm{mg} / \mathrm{kg} \mathrm{MHC}$ groups on day $42(p>0.05)$. 
Table 4. Effect of dietary supplementation with manganese hydroxychloride on slaughter performance of broilers at the age of day $42(\%)^{1}$.

\begin{tabular}{|c|c|c|c|c|c|c|c|c|c|c|c|}
\hline \multirow{2}{*}{ Item } & \multirow{2}{*}{$\begin{array}{c}\begin{array}{c}\mathrm{Mn} \text { as } \\
\mathrm{MnSO}_{4} \\
(\mathrm{mg} / \mathrm{kg})\end{array} \\
100\end{array}$} & \multicolumn{6}{|c|}{ Mn as Manganese Hydroxychloride (mg/kg) } & \multirow{2}{*}{ SEM } & \multicolumn{3}{|c|}{$p$-Value } \\
\hline & & 0 & 20 & 40 & 60 & 80 & 100 & & ANOVA & Linear & Quadratic \\
\hline $\begin{array}{c}\text { Carcass } \\
\text { weight/body } \\
\text { weight }\end{array}$ & 92.24 & 91.09 & 91.57 & 91.18 & 91.74 & 91.60 & 92.54 & 0.454 & 0.296 & 0.048 & 0.442 \\
\hline $\begin{array}{l}\text { Eviscerated } \\
\text { weight/body } \\
\text { weight }\end{array}$ & 69.72 & 69.04 & 70.21 & 70.14 & 71.45 & 70.82 & 70.58 & 0.639 & 0.101 & 0.057 & 0.130 \\
\hline $\begin{array}{c}\text { Breast } \\
\text { weight/eviscerated } \\
\text { weight }\end{array}$ & 25.04 & 25.76 & 25.42 & 25.08 & 25.90 & 24.27 & 25.98 & 0.622 & 0.473 & 0.777 & 0.382 \\
\hline $\begin{array}{c}\text { Leg } \\
\text { weight/eviscerated } \\
\text { weight }\end{array}$ & 22.31 & 22.21 & 21.74 & 21.35 & 21.65 & 21.73 & 22.26 & 0.632 & 0.927 & 0.921 & 0.261 \\
\hline $\begin{array}{c}\text { Abdominal fat } \\
\text { weight/eviscerated } \\
\text { weight }\end{array}$ & 1.34 & 1.53 & 1.43 & 1.31 & 1.40 & 1.38 & 1.42 & 0.143 & 0.925 & 0.589 & 0.376 \\
\hline
\end{tabular}

${ }^{1}$ Values represent the means of six pens $(n=6)$ per treatment.

Table 5. Effect of dietary supplementation with manganese hydroxychloride on antioxidant capacity in serum of broilers ${ }^{1}$.

\begin{tabular}{|c|c|c|c|c|c|c|c|c|c|c|c|}
\hline \multirow{2}{*}{ Item $^{2}$} & \multirow{2}{*}{$\begin{array}{c}\begin{array}{c}\mathrm{Mn} \text { as } \\
\mathrm{MnSO}_{4} \\
(\mathrm{mg} / \mathrm{kg})\end{array} \\
100\end{array}$} & \multicolumn{6}{|c|}{ Mn as Manganese Hydroxychloride (mg/kg) } & \multirow{2}{*}{ SEM } & \multicolumn{3}{|c|}{$p$-Value } \\
\hline & & 0 & 20 & 40 & 60 & 80 & 100 & & ANOVA & Linear & Quadratic \\
\hline \multicolumn{12}{|l|}{ Day 21} \\
\hline $\begin{array}{c}\text { CAT } \\
(\mathrm{U} / \mathrm{mL})\end{array}$ & $67.21^{\mathrm{a}}$ & $48.37^{b}$ & $61.12^{\mathrm{a}}$ & $\begin{array}{c}64.13 \\
a\end{array}$ & $64.33^{\mathrm{a}}$ & $64.12^{\mathrm{a}}$ & $\begin{array}{c}64.52 \\
a\end{array}$ & 2.823 & 0.001 & $<0.001$ & 0.052 \\
\hline $\begin{array}{l}\text { MnSOD } \\
(\mathrm{U} / \mathrm{mL})\end{array}$ & $54.19^{\text {a }}$ & $29.23^{b}$ & $34.79^{\mathrm{b}}$ & $\begin{array}{c}39.87 \\
b\end{array}$ & $40.29^{b}$ & $56.05^{\mathrm{a}}$ & $\begin{array}{c}57.44 \\
a\end{array}$ & 4.589 & $<0.001$ & $<0.001$ & 0.610 \\
\hline $\begin{array}{c}\text { MDA } \\
(\mathrm{nmol} / \mathrm{mL})\end{array}$ & 4.24 & 5.26 & 5.22 & 4.63 & 4.49 & 4.22 & 4.08 & 0.299 & 0.055 & 0.002 & 0.782 \\
\hline $\begin{array}{l}\text { T-AOC } \\
(\mathrm{U} / \mathrm{mL})\end{array}$ & 11.04 & 10.75 & 10.60 & 11.03 & 10.67 & 10.72 & 10.69 & 0.217 & 0.369 & 0.894 & 0.666 \\
\hline \multicolumn{12}{|l|}{ Day 42} \\
\hline $\begin{array}{c}\text { CAT } \\
(\mathrm{U} / \mathrm{mL})\end{array}$ & $66.65^{b}$ & $52.89^{\mathrm{c}}$ & $66.08^{b}$ & $\begin{array}{c}66.98 \\
b\end{array}$ & $67.29^{b}$ & $78.28^{a}$ & $\begin{array}{c}75.89 \\
a\end{array}$ & 1.755 & $<0.001$ & $<0.001$ & 0.027 \\
\hline $\begin{array}{l}\text { MnSOD } \\
(\mathrm{U} / \mathrm{mL})\end{array}$ & $25.59^{b}$ & $24.14^{\mathrm{c}}$ & $30.12^{b}$ & $\begin{array}{c}32.93 \\
\mathrm{ab}\end{array}$ & $33.13^{a b}$ & $36.88^{a}$ & $\begin{array}{c}32.86 \\
a b\end{array}$ & 1.431 & $<0.001$ & $<0.001$ & 0.002 \\
\hline $\begin{array}{c}\text { MDA } \\
(\mathrm{nmol} / \mathrm{mL})\end{array}$ & $4.11^{\mathrm{c}}$ & $5.75^{\mathrm{a}}$ & $5.16^{b}$ & $\begin{array}{l}4.67 \\
\mathrm{bc}\end{array}$ & $4.78^{b c}$ & $4.60^{b c}$ & $4.15^{c}$ & 0.196 & $<0.001$ & $<0.001$ & 0.233 \\
\hline $\begin{array}{l}\text { T-AOC } \\
(\mathrm{U} / \mathrm{mL})\end{array}$ & $11.68^{\mathrm{b}}$ & $8.71^{\mathrm{d}}$ & $10.09^{c}$ & $\underset{\mathrm{b}}{11.11}$ & $11.12^{b}$ & $12.92^{\mathrm{a}}$ & $\begin{array}{c}12.02 \\
\mathrm{ab}\end{array}$ & 0.275 & $<0.001$ & $<0.001$ & 0.012 \\
\hline
\end{tabular}

${ }^{1}$ Values represent the means of six pens $(n=6)$ per treatment. ${ }^{2}$ CAT, Catalase; MnSOD, Manganese superoxide dismutase; MDA, Malonaldehyde; T-AOC, Total antioxidant capacity. ${ }^{a, b, c}$ Means within a row with different superscripts are significantly different at the $p$-value indicated for ANOVA.

As shown in Table 6, diet supplemented with $\mathrm{MnSO}_{4}$ or $80-100 \mathrm{mg} / \mathrm{kg}$ Mn as MHC increased MnSOD activity of liver on day $21(p<0.05)$ compared to that of control group. With the increasing of dietary MHC supplementation level, the activities of MnSOD (linear, $p<0.05$; quadratic, $p<0.05$ ) and CAT (linear, $p<0.05$ ), and T-AOC (linear, $p<0.05$; quadratic, $p<0.05$ ) of liver increased on day 42 , while MDA level decreased (linear, $p<0.05)$. Additionally, CAT activity and T-AOC with $60 \mathrm{mg} / \mathrm{kg}$ MHC treatment were greater than those in the $\mathrm{MnSO}_{4}$ treatment $(p<0.05)$. 
Table 6. Effect of dietary supplementation with manganese hydroxychloride on antioxidant capacity in liver of broilers ${ }^{1}$.

\begin{tabular}{|c|c|c|c|c|c|c|c|c|c|c|c|}
\hline \multirow{2}{*}{ Item $^{2}$} & \multirow{2}{*}{$\begin{array}{c}\begin{array}{c}\mathrm{Mn} \text { as } \\
\mathrm{MnSO}_{4} \\
(\mathrm{mg} / \mathrm{kg})\end{array} \\
100\end{array}$} & \multicolumn{6}{|c|}{ Mn as Manganese Hydroxychloride (mg/kg) } & \multirow{2}{*}{ SEM } & \multicolumn{3}{|c|}{$p$-Value } \\
\hline & & 0 & 20 & 40 & 60 & 80 & 100 & & ANOVA & Linear & Quadratic \\
\hline \multicolumn{12}{|l|}{ Day 21} \\
\hline $\begin{array}{l}(\mathrm{U} / \mathrm{mg} \\
\text { protein) }\end{array}$ & 4.17 & 3.02 & 3.30 & 3.45 & 3.26 & 3.59 & 3.93 & 0.332 & 0.220 & 0.057 & 0.723 \\
\hline $\begin{array}{l}\text { MnSOD } \\
\text { (U/mg } \\
\text { protein) }\end{array}$ & $4.94^{\mathrm{ab}}$ & $2.68^{\mathrm{c}}$ & $3.38 \mathrm{bc}$ & $\begin{array}{l}4.05 \\
\mathrm{abc}\end{array}$ & $4.33^{a b c}$ & $4.46^{\mathrm{ab}}$ & $5.58^{\mathrm{a}}$ & 0.537 & 0.013 & $<0.001$ & 0.995 \\
\hline $\begin{array}{c}\text { MDA } \\
\text { (nmol/mg } \\
\text { protein) }\end{array}$ & 0.36 & 0.49 & 0.49 & 0.47 & 0.44 & 0.48 & 0.40 & 0.037 & 0.145 & 0.097 & 0.675 \\
\hline $\begin{array}{l}\text { T-AOC } \\
\text { (U/mg } \\
\text { protein) }\end{array}$ & 1.46 & 1.39 & 1.46 & 1.44 & 1.48 & 1.25 & 1.39 & 0.075 & 0.355 & 0.313 & 0.432 \\
\hline Day 42 & & & & & & & & & & & \\
\hline $\begin{array}{c}\text { CAT } \\
\text { (U/mg } \\
\text { protein) }\end{array}$ & $4.48^{b c}$ & $3.96^{\mathrm{c}}$ & $4.50^{b c}$ & $\begin{array}{l}4.56 \\
\mathrm{bc}\end{array}$ & $5.22^{\mathrm{a}}$ & $4.74^{\mathrm{ab}}$ & $\begin{array}{l}4.66 \\
\mathrm{ab}\end{array}$ & 0.199 & 0.008 & 0.007 & 0.084 \\
\hline $\begin{array}{l}\text { MnSOD } \\
\text { (U/mg } \\
\text { protein) }\end{array}$ & $4.11^{\mathrm{a}}$ & $2.87^{b}$ & $4.48^{\mathrm{a}}$ & $4.27^{\mathrm{a}}$ & $4.77^{\mathrm{a}}$ & $4.14^{\mathrm{a}}$ & $4.05^{\mathrm{a}}$ & 0.290 & 0.004 & 0.021 & 0.002 \\
\hline $\begin{array}{c}\text { MDA } \\
\text { (nmol/mg } \\
\text { protein) }\end{array}$ & $0.47^{\mathrm{ab}}$ & $0.55^{\mathrm{a}}$ & $0.49^{a b}$ & $\begin{array}{l}0.48 \\
\mathrm{ab}\end{array}$ & $0.42^{b}$ & $0.41^{b}$ & $0.44^{b}$ & 0.028 & 0.018 & 0.001 & 0.103 \\
\hline $\begin{array}{l}\text { T-AOC } \\
\text { (U/mg } \\
\text { protein) }\end{array}$ & $1.24^{\mathrm{b}}$ & $1.07^{\mathrm{c}}$ & $1.54^{\mathrm{ab}}$ & $1.64^{a}$ & $1.73^{\mathrm{a}}$ & $1.61^{\mathrm{a}}$ & $\begin{array}{c}1.53 \\
\mathrm{ab}\end{array}$ & 0.113 & 0.002 & 0.015 & 0.003 \\
\hline
\end{tabular}

${ }^{1}$ Values represent the means of six pens $(n=6)$ per treatment. ${ }^{2}$ CAT, Catalase; MnSOD, Manganese superoxide dismutase; MDA, Malonaldehyde; T-AOC, Total antioxidant capacity. ${ }^{a, b, c}$ Means within a row with different superscripts are significantly different at the $p$-value indicated for ANOVA.

\subsection{Tibial Parameters}

Effects of dietary supplementation with MHC on tibial parameters of broilers are presented in Table 7. With the increase of MHC supplementation level, the length, strength, and density index of tibia increased linearly on day $21(p<0.05)$. However, there were no significant differences in all measured tibial parameters among treatments were detected on day $42(p>0.05)$. Additionally, no differences in tibial parameters between $\mathrm{MnSO}_{4}$ group and 40-100 mg/kg MHC groups were observed $(p>0.05)$.

\subsection{Mn Contents in Tissues}

As shown in Table 8, the contents of Mn in heart, liver, kidney, and tibia of broilers increased linearly on day $42(p<0.05)$ as supplemental MHC level increased. However, there was no difference in Mn level of serum and chicken breast muscle among all groups were detected $(p>0.05)$. Additionally, no differences in the Mn contents in liver and tibia of broilers between $\mathrm{MnSO}_{4}$ and $80 \mathrm{mg} / \mathrm{kg}$ MHC group were observed $(p>0.05)$. 
Table 7. Effect of dietary supplementation with manganese hydroxychloride on tibia parameters of broilers ${ }^{1}$.

\begin{tabular}{|c|c|c|c|c|c|c|c|c|c|c|c|}
\hline \multirow{2}{*}{ Item } & \multirow{2}{*}{$\begin{array}{c}\begin{array}{c}\mathrm{Mn} \text { as } \\
\mathrm{MnSO}_{4} \\
(\mathrm{mg} / \mathrm{kg})\end{array} \\
100\end{array}$} & \multicolumn{6}{|c|}{ Mn as Manganese Hydroxychloride (mg/kg) } & \multirow{2}{*}{ SEM } & \multicolumn{3}{|c|}{$p$-Value } \\
\hline & & $\mathbf{0}$ & 20 & 40 & 60 & 80 & 100 & & ANOVA & Linear & Quadratic \\
\hline \multicolumn{12}{|l|}{ Day 21} \\
\hline Weight (g) & 6.81 & 6.33 & 6.35 & 6.54 & 6.60 & 6.56 & 6.55 & 0.231 & 0.806 & 0.369 & 0.628 \\
\hline $\begin{array}{l}\text { Length } \\
(\mathrm{mm})\end{array}$ & $76.07^{\mathrm{a}}$ & $71.96^{c}$ & $72.35^{b c}$ & $\begin{array}{l}74.43 \\
\mathrm{ab}\end{array}$ & $75.29^{a}$ & $76.12^{\mathrm{a}}$ & $\begin{array}{c}75.17 \\
\mathrm{a}\end{array}$ & 0.754 & 0.001 & $<0.001$ & 0.172 \\
\hline $\begin{array}{l}\text { Diameter } \\
(\mathrm{mm})\end{array}$ & 6.08 & 5.76 & 5.85 & 6.14 & 6.27 & 6.21 & 6.18 & 0.172 & 0.297 & 0.141 & 0.235 \\
\hline $\begin{array}{l}\text { Strength } \\
\text { (N) }\end{array}$ & 132.72 & 124.19 & 123.30 & 129.78 & 128.11 & 130.02 & 132.31 & 2.865 & 0.159 & 0.025 & 0.932 \\
\hline $\begin{array}{c}\text { Density } \\
\text { index } \\
\left(\mathrm{g} / \mathrm{cm}^{2}\right) \\
\text { Day } 42\end{array}$ & $0.21^{\mathrm{a}}$ & $0.19^{b}$ & $0.19^{b}$ & $\begin{array}{c}0.20 \\
\mathrm{ab}\end{array}$ & $0.20^{a b}$ & $0.20^{a b}$ & $0.21^{\mathrm{a}}$ & 0.005 & 0.035 & 0.006 & 0.965 \\
\hline Weight (g) & 19.37 & 18.82 & 18.40 & 18.40 & 17.86 & 19.68 & 18.19 & 0.609 & 0.358 & 0.975 & 0.739 \\
\hline $\begin{array}{l}\text { Length } \\
(\mathrm{mm})\end{array}$ & 111.40 & 109.25 & 109.16 & 108.25 & 108.89 & 111.21 & 108.61 & 0.976 & 0.169 & 0.635 & 0.961 \\
\hline $\begin{array}{l}\text { Diameter } \\
(\mathrm{mm})\end{array}$ & 9.59 & 10.37 & 9.08 & 9.54 & 9.19 & 9.87 & 9.66 & 0.417 & 0.398 & 0.678 & 0.136 \\
\hline $\begin{array}{l}\text { Strength } \\
\text { (N) }\end{array}$ & 316.65 & 295.13 & 295.77 & 301.15 & 307.36 & 309.22 & 317.97 & 7.460 & 0.181 & 0.017 & 0.706 \\
\hline $\begin{array}{l}\text { Density } \\
\text { index } \\
\left(\mathrm{g} / \mathrm{cm}^{2}\right)\end{array}$ & 0.30 & 0.29 & 0.28 & 0.29 & 0.29 & 0.29 & 0.29 & 0.005 & 0.807 & 0.829 & 0.769 \\
\hline
\end{tabular}

${ }^{1}$ Values represent the means of six pens $(n=6)$ per treatment. ${ }^{\mathrm{a}, \mathrm{b}, \mathrm{c}}$ Means within a row with different superscripts are significantly different at the $p$-value indicated for ANOVA.

Table 8. Effect of dietary supplementation with manganese hydroxychloride on Mn content in tissue of broilers on day $42\left(\mathrm{mg} / \mathrm{kg}\right.$, dry matter basis) ${ }^{1}$.

\begin{tabular}{|c|c|c|c|c|c|c|c|c|c|c|c|}
\hline \multirow{2}{*}{ Item } & \multirow{2}{*}{$\begin{array}{c}\begin{array}{c}\mathrm{Mn} \text { as } \\
\mathrm{MnSO}_{4} \\
(\mathrm{mg} / \mathrm{kg})\end{array} \\
100\end{array}$} & \multicolumn{6}{|c|}{ Mn as Manganese Hydroxychloride (mg/kg) } & \multirow{2}{*}{ SEM } & \multicolumn{3}{|c|}{$p$-Value } \\
\hline & & 0 & 20 & 40 & 60 & 80 & 100 & & ANOVA & Linear & Quadratic \\
\hline Serum & 4.30 & 4.25 & 5.40 & 5.16 & 5.11 & 5.82 & 5.24 & 0.511 & 0.305 & 0.293 & 0.185 \\
\hline Heart & $1.06^{\mathrm{a}}$ & $0.64^{b}$ & $0.71^{b}$ & $0.70^{b}$ & $0.77^{b}$ & $0.79^{b}$ & $1.05^{\mathrm{a}}$ & 0.065 & $<0.001$ & $<0.001$ & 0.131 \\
\hline Liver & $10.40^{\mathrm{ab}}$ & $9.16^{b}$ & $9.43^{b}$ & $9.59^{b}$ & $10.55^{\mathrm{ab}}$ & $11.60^{a}$ & $\begin{array}{c}11.66 \\
a\end{array}$ & 0.577 & 0.015 & 0.001 & 0.668 \\
\hline $\begin{array}{l}\text { Breast } \\
\text { muscle }\end{array}$ & 0.42 & 0.37 & 0.38 & 0.40 & 0.41 & 0.41 & 0.39 & 0.023 & 0.703 & 0.371 & 0.205 \\
\hline Kidney & $7.80^{\mathrm{a}}$ & $5.68^{d}$ & $6.36^{c}$ & $\begin{array}{c}6.76 \\
\mathrm{bc}\end{array}$ & $6.80^{b c}$ & $6.83^{b c}$ & $\begin{array}{c}7.24 \\
\mathrm{ab}\end{array}$ & 0.230 & $<0.001$ & $<0.001$ & 0.008 \\
\hline Tibia & $10.22^{\mathrm{a}}$ & $4.74^{\mathrm{c}}$ & $5.75^{c}$ & $5.90^{\mathrm{c}}$ & $8.24^{b}$ & $8.36^{a b}$ & $\begin{array}{c}8.39 \\
a b\end{array}$ & 0.619 & $<0.001$ & $<0.001$ & 0.375 \\
\hline
\end{tabular}

${ }^{1}$ Values represent the means of six pens $(n=6)$ per treatment. ${ }^{\text {a-d }}$ Means within a row with different superscripts are significantly different at the $p$-value indicated for ANOVA.

\subsection{The Optimal Supplementation Level}

According to the quadratic regression curve of MnSOD activities to dietary MHC level in serum and liver on day 42 (Figure 1), the optimal dietary supplementation level of $\mathrm{Mn}$ in the form of MHC is $72.18 \mathrm{mg} / \mathrm{kg}$ and $60.18 \mathrm{mg} / \mathrm{kg}$, respectively; and the suitable inclusion level is $53.77-90.58 \mathrm{mg} / \mathrm{kg}$ and $49.88-70.47 \mathrm{mg} / \mathrm{kg}$, respectively. 


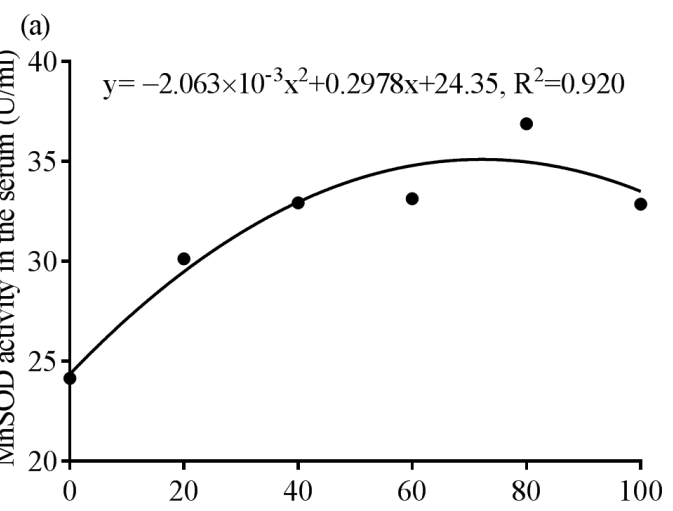

Dietary Mn as Mn hydroxychloride supplementation level ( $\mathrm{mg} / \mathrm{kg})$

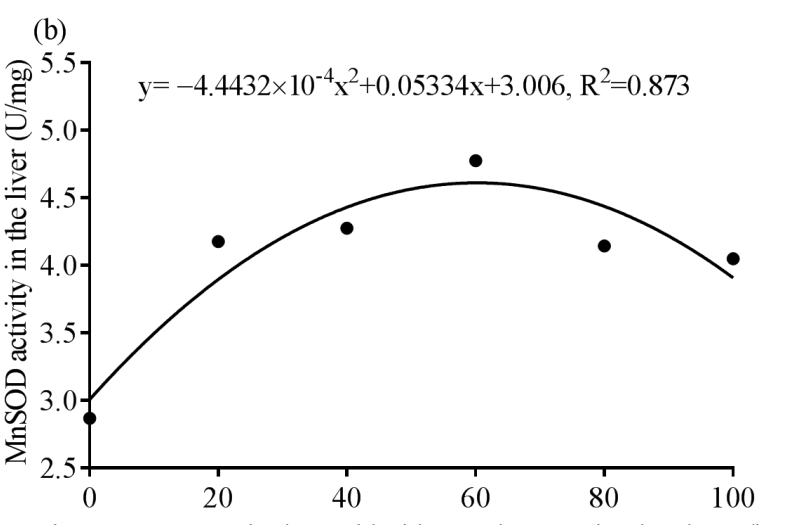

Dietary Mn as Mn hydroxychloride supplementation level (mg/kg)

Figure 1. The quadratic fit model of dietary manganese (Mn) supplementation level as Mn hydroxychloride (MHC) and Mn superoxide dismutase (MnSOD) activities in the serum (a) and liver (b) of broilers on day 42.

For MnSOD activity in the serum, the quadratic model was $y=-2.063 \times 10^{-3} x^{2}+0.2978 x+24.35$, $\mathrm{R}^{2}=0.920, p=0.023$, the best addition level of $\mathrm{Mn}$ as MHC is $72.18 \mathrm{mg} / \mathrm{kg}$, and the optimal addition range is $53.77-90.58 \mathrm{mg} / \mathrm{kg}$. For MnSOD activity in the liver, the quadratic model was $y=-4.4432 \times 10^{-4} x^{2}+0.05334 x+3.006, \mathrm{R}^{2}=0.873, p=0.046$, the best addition level of $\mathrm{Mn}$ as $\mathrm{MHC}$ is $60.18 \mathrm{mg} / \mathrm{kg}$, and the optimal addition range is $49.88-70.47 \mathrm{mg} / \mathrm{kg}$.

\section{Discussion}

The reports related to the effects of Mn on growth performance of broilers were inconsistent. Many studies have shown that dietary supplementation with different chemical forms of Mn such as Mn propionate [12], Mn proteinates [23], Mn oxide [24], Mn sulfate [25], Mn fumarate [26], or Mn amino acid chelate [27] did not significantly affect ADG, ADFI, and F:G of broilers. However, Meng et al. [28] found dietary inclusion of $50 \mathrm{mg} / \mathrm{kg}$ Mn as Mn methionine hydroxyl analog chelated could improve ADG and ADFI of broilers. Otherwise, Ognik et al. [29] reported that diet supplemented with 50 or $100 \mathrm{mg} / \mathrm{kg} \mathrm{Mn}$ in the form of Mn oxide nanoparticles decreased F:G of turkeys. At present, there are a few studies on the effect of MHC on broiler, and the results are inconsistent. Conly et al. [30] showed that diet (45 mg/kg Mn) supplemented with 30-130 mg/kg Mn in the form of MHC had no significant effect on feed intake, body weight, and F:G of Cobb 500 broilers. The present study also showed that dietary (37 mg/kg Mn) supplementation of $20-100 \mathrm{mg} / \mathrm{kg} \mathrm{Mn}$ as MHC had no significant effect on the ADG, ADFI, and F:G of AA broilers. However, Jasek et al. [16] reported dietary (40 mg/kg Mn) inclusion of 40-160 mg/kg Mn as MHC decreased F:G of Ross 708 broilers. These disparities in results among studies may be due to the difference in experiment broiler breed, Mn content in basal diet, source and addition level of Mn.

Carcass characteristics are important parameters for evaluating the meat production performance of broilers. Studies have shown that dietary supplementation of $100 \mathrm{mg} / \mathrm{kg}$ $\mathrm{Mn}$ as $\mathrm{MnO}$ and $\mathrm{Mn}_{2} \mathrm{O}_{3}$ nanoparticles improved the carcass yield of turkeys [31], and supplementation of $100 \mathrm{mg} / \mathrm{kg} \mathrm{Mn}$ in the form of $\mathrm{MnSO}_{4}$ or amino acid chelated $\mathrm{Mn}$ reduced the abdominal fat rate of broilers [25,32,33]. The present study showed that dietary inclusion of MHC did not significantly affect slaughter characteristics of broilers, and there were no differences with $\mathrm{MnSO}_{4}$ were detected. Matuszewski et al. [34] also reported that dietary supplementation with $\mathrm{Mn}_{2} \mathrm{O}_{3}$ and $\mathrm{Mn}_{2} \mathrm{O}_{3}$ nanoparticles (21-70 mg/kg) did not significantly affect any slaughter characteristics of broilers. Further experiments need to be conducted with several Mn sources and levels under the same condition, especially in large-scale commercial farm conditions to confirm the results.

Parameter CAT, T-AOC, and MDA are usually used to evaluate the antioxidant ability of animals. As a component of MnSOD, Mn can improve broiler's antioxidant ability by catalyzing the reduction of superoxide anion to hydrogen peroxide [35]. An in vitro study 
indicated that MnSOD activity and mRNA expression level in chick embryonic myocardial cells were improved by $1.0 \mathrm{mmol} / \mathrm{L}$ of $\mathrm{Mn}$ as $\mathrm{MnCl}_{2}$ treatment [36]. Studies also have shown that dietary inclusion of $\mathrm{Mn}$ as $\mathrm{MnSO}_{4}, \mathrm{Mn}$ methionine, or Mn oxide improved MnSOD, CAT, and GSH-Px activities and T-AOC, while reducing MDA level in serum, liver, and leg muscle of broilers [25,37]. In this study, dietary inclusion of MHC increased MnSOD and CAT activities and T-AOC, while decreasing MDA content in serum and liver of broilers. Therefore, dietary supplementation with MHC can improve the antioxidant capacity and reduce oxidative damage of broilers by improving antioxidative enzyme activities and reducing peroxidation products content. The increase of MnSOD activity may be due to that Mn activating protein kinase $\mathrm{C}$ and protein tyrosine kinase [35], altering specificity protein 1 and activating protein-2 DNA-binding activities, and enhancing MnSOD binding protein RNA-binding activity at the translational level [38]. The increase of other antioxidant enzymes may be related to the activation of Nrf2 signaling pathway by Mn treatment [39]. Additionally, due to the MnSOD activities in serum and liver showing significant quadratic response to dietary MHC addition level, it can be also concluded that the optimal dietary supplementation level of $\mathrm{Mn}$ in the form of MHC is $50-90 \mathrm{mg} / \mathrm{kg}$ according to the quadratic regression curve of MnSOD activities in the serum and liver. The broken-line models are not shown here because the data better fitted the quadratic model $\left(R^{2}=0.920\right.$ and 0.873 for the serum and liver, respectively) than a broken line $\left(R^{2}=0.820\right.$ and 0.844 for the serum and liver, respectively).

Studies showed that Mn deficiency experimental model of broilers was successfully established at the dose of $40 \mathrm{mg} / \mathrm{kg}$ which can affect the normal development of tibia by inhibiting the vitality of osteoblasts and chondrocyte proliferation and promoting chondrocyte apoptosis in the tibia $[40,41]$, disordering the level of bone regulatory hormones and enzymes of bone metabolism in the serum [42], and leading to metaphyseal osteoporosis [40]. The length, weight, diameter, breaking strength, or density index of tibia are usual parameters to be used for evaluating development of tibia. Studies reported that dietary supplementation of $\mathrm{Mn}$ as $\mathrm{MnSO}_{4}, \mathrm{MnCO}_{3}$, and $\mathrm{MnO}$ could increase the length, weight, diameter, breaking strength, and density index of tibia in broilers [42-44], reduce the incidence of leg abnormalities [25,32]. However, Bozkurt et al. [37] reported that dietary supplementation of $\mathrm{Mn}$ as $\mathrm{Mn}$-methionine and $\mathrm{MnO}$ with levels $12.5,25$, and $50 \mathrm{mg} / \mathrm{kg}$ has no effect on the weight, length, diameter, and density index of tibia in broilers. It is assumed that the different bioavailability of different chemical forms in Mn sources may be the reason which resulted in these inconsistent results. In this study, it is shown that dietary supplementation of Mn as MHC increased length and density index of tibia in the early growth stage of broilers, which may be due to that broilers had low feed intake, and rapid bone growth and development, especially during the first two week of post-hatch age when the bone is not completely formed [45]; whereas, broilers can obtain sufficient Mn for bone growth due to the increase of feed intake at the late growth stage.

The source and addition level of Mn in diet may directly affect the Mn content of broiler tissue. Dietary supplementation of $\mathrm{Mn}$ in the form of $\mathrm{MnSO}_{4}, \mathrm{MnO}$, or $\mathrm{Mn}$ fumarate could improve Mn levels in tibia, liver, and kidney of broilers [26,46]. In the present study, dietary supplementation with MHC improved Mn levels in the heart, liver, kidney, and tibia, which was agreed with the study conducted by Conly et al. [30]. However, it is also found that dietary Mn level has no significant effect on the Mn content in serum and breast muscle. European Food Safety Authority (2016) also reported that dietary MHC or $\mathrm{MnSO}_{4}$ increased Mn levels in the liver and tibia but did not significantly affect Mn level in breast muscle [14]. This may be due to the weak ability to deposit Mn in breast muscle, where mitochondria are not abundant. And most of $\mathrm{Mn}$ in serum was transferred to other organizations.

According to the results of the present study, it seems that the efficacy of MHC is a little higher than that of $\mathrm{MnSO}_{4}$ on the basis of some measured indicators including antioxidant capacity, tibial parameters, and Mn contents in liver and tibia. So it is assumed that MHC maybe have higher relative bioavailability than that of $\mathrm{MnSO}_{4}$. MHC was combined by 
covalent bonds between $\mathrm{Mn}$, hydroxy groups as well as chloride ions, creating a stronger chemical bond than traditional sulfate minerals [14]. The covalent bonds possessed by hydroxychloride minerals can also reduce the reaction of minerals with other components in feeds [47], so its bioavailability could be potentially improved. However, the accurate relative biological availability of $\mathrm{Mn}$ as MHC to Mn sulfate has not been reported, which needs to be further studied.

\section{Conclusions}

Dietary inclusion of MHC can improve the antioxidant capacity, bone quality, and Mn deposition of broilers, but no effects on growth performance were detected. Dietary inclusion of $50-90 \mathrm{mg} / \mathrm{kg} \mathrm{Mn}$ as MHC is recommended in broilers.

Author Contributions: Conceptualization, Y.S. and L.Z.; Data curation, Y.S.; Formal analysis, Y.S.; Funding acquisition, L.Z. Investigation, S.G.; Methodology, Y.S.; Project administration, L.Z.; Resources, T.Y., Y.L., Y.Z. and Y.D.; Software, Y.S.; Supervision, J.L.; Validation, Y.S. and L.Z.; Visualization, Y.S. and S.G.; Writing—original draft, Y.S.; Writing-review \& editing, J.L. and L.Z. All authors have read and agreed to the published version of the manuscript.

Funding: This research was funded by Ministry of Agriculture and Rural Areas Feed Industry Centre (MAFIC), grant number 201805410410599, and the APC was funded by MAFIC.

Institutional Review Board Statement: The study was conducted according to the guidelines of the Declaration of Helsinki, and approved by the Institutional Animal Care and Use Committee of China Agricultural University (approval code: AW30801202-11; 3 August 2021).

Informed Consent Statement: Not applicable.

Data Availability Statement: The data presented in this study are available on request from the corresponding author.

Acknowledgments: The authors gratefully acknowledge the help of Miaomiao Han, Wenqing Lai, Dongting He, Chi Zhang, Wenwei Zhang, Jiaren Ding, and Chaoyi Chi of Ministry of Agriculture and Rural Areas Feed Industry Centre.

Conflicts of Interest: The authors declare no conflict of interest.

\section{References}

1. Spears, J.W. Boron, chromium, manganese, and nickel in agricultural animal production. Biol. Trace Elem. Res. 2019, 188, 35-44. [CrossRef]

2. Hassan, S.; Hassan, F.U.; Rehman, M.S.U. Nano-particles of trace minerals in poultry nutrition: Potential applications and future prospects. Biol. Trace Elem. Res. 2020, 195, 591-612. [CrossRef] [PubMed]

3. Mwangi, S.; Timmons, J.; Ao, T.; Paul, M.; Macalintal, L.; Pescatore, A.; Cantor, A.; Dawson, K.A. Effect of manganese preconditioning and replacing inorganic manganese with organic manganese on performance of male broiler chicks. Poult. Sci. 2018, 98, 2105-2113. [CrossRef]

4. Patra, A.; Lalhriatpuii, M. progress and prospect of essential mineral nanoparticles in poultry nutrition and feeding-A review. Biol. Trace Elem. Res. 2020, 197, 233-253. [CrossRef] [PubMed]

5. Wang, C.; Guan, Y.; Lv, M.; Zhang, R.; Guo, Z.; Wei, X.; Du, X.; Yang, J.; Li, T.; Wan, Y.; et al. Manganese increases the sensitivity of the cGAS-STING pathway for double-stranded DNA and is required for the host defense against DNA viruses. Immunity 2018, 48, 675-687. [CrossRef]

6. Pan, S.; Zhang, K.; Ding, X.; Wang, J.; Peng, H.; Zeng, Q.; Xuan, Y.; Su, Z.; Wu, B.; Bai, S. Effect of high dietary manganese on the immune responses of broilers following oral salmonella typhimurium inoculation. Biol. Trace Elem. Res. 2018, 181, 347-360. [CrossRef]

7. National Research Council (NRC). Nutrient Requirements of Poultry, 9th ed.; National Academy Press: Washington, DC, USA, 1994; pp. 57-63.

8. Halpin, K.M.; Chausow, D.G.; Baker, D.H. Efficiency of manganese absorption in chicks fed corn-soy and casein diets. J. Nutr. 1986, 116, 1747-1751. [CrossRef]

9. Tufarelli, V.; Laudadio, V. Manganese and its role in poultry nutrition: An overview. J. Exp. Biol. Agr. Sci. 2017, 5, 749-754. [CrossRef]

10. Yenice, E.; Mizrak, C.; Gültekin, M.; Atik, Z.; Tunca, M. Effects of organic and inorganic forms of manganese, zinc, copper, and chromium on bioavailability of these minerals and calcium in late-phase laying hens. Biol. Trace Elem. Res. 2015, 167, 300-307. [CrossRef] [PubMed] 
11. Wang, F.; Lu, L.; Li, S.; Liu, S.; Zhang, L.; Yao, J.; Luo, X. Relative bioavailability of manganese proteinate for broilers fed a conventional corn-soybean meal diet. Biol. Trace Elem. Res. 2012, 146, 181-186. [CrossRef] [PubMed]

12. Brooks, M.A.; Grimes, J.L.; Lloyd, K.E.; Valdez, F.; Spears, J.W. Relative bioavailability in chicks of manganese from manganese propionate. J. Appl. Poult. Res. 2012, 21, 126-130. [CrossRef]

13. Wang, Y.S.; Zhang, J.P.; Gang, Y. Solubility and phase diagrams of hydroxyl manganese chloride. Trans. Nonferrous Met. Soc. 2011, 21, 1136-1140. [CrossRef]

14. European Food Safety Authority. Safety and efficacy of manganese hydroxychloride as feed additive for all animal species. EFSA J. 2016, 14, e04474.

15. Jasek, A.; Parr, T.; Coufal, C.; Lee, J. Research note: Evaluation of manganese hydroxychloride in 45-wk-old white leghorn layers using yolk and shell manganese content. Poult. Sci. 2020, 99, 1084-1087. [CrossRef]

16. Jasek, A.; Coufal, C.; Parr, T.; Lee, J. Evaluation of Increasing Manganese Hydroxychloride Level on Male Broiler Growth Performance and Tibia Strength. J. Appl. Poult. Res. 2019, 28, 1039-1047. [CrossRef]

17. Kerkaert, H.; Woodworth, J.; DeRouchey, J.; Dritz, S.; Tokach, M.; Goodband, R.; Manzke, N. Determining the effects of manganese source and level in diets containing high levels of copper on growth performance of growing-finishing pigs. Kans. Agric. Exp. Stn. Res. Rep. 2020, 6, 19. [CrossRef]

18. Ghazalah, A.; Abd-Elsamee, M.; Ibrahim, M.; Abdelgayed, S.S.; Abdelkader, M.; Gonzalez-Sanchez, D.; Wealleans, A. Effects of a combination of lysolecithin, synthetic emulsifier, and monoglycerides on growth performance, intestinal morphology, and selected carcass traits in broilers fed low-energy diets. Animals 2021, 11, 3037. [CrossRef]

19. Riahi, I.; Ramos, A.J.; Raj, J.; Jakovčević, Z.; Farkaš, H.; Vasiljević, M.; Pérez-Vendrell, A.M. Effect of a mycotoxin binder (MMDA) on the growth performance, blood and carcass characteristics of broilers fed ochratoxin a and $\mathrm{t}-2$ mycotoxin contaminated diets. Animals 2021, 11, 3205. [CrossRef] [PubMed]

20. Meng, Y.; Huo, H.; Zhang, Y.; Bai, S.; Wang, R.; Zhang, K.; Ding, X.; Wang, J.; Zeng, Q.; Peng, H.; et al. effects of dietary glucose oxidase supplementation on the performance, apparent ileal amino acids digestibility, and ileal microbiota of broiler chickens. Animals 2021, 11, 2909. [CrossRef] [PubMed]

21. Domínguez-Negrete, A.; Gómez-Rosales, S.; Angeles, M.d.L.; López-Hernández, L.H.; Reis de Souza, T.C.; Latorre-Cárdenas, J.D.; Téllez-Isaias, G. Addition of different levels of humic substances extracted from worm compost in broiler feeds. Animals 2021, 11, 3199. [CrossRef]

22. Sarker, M.T.; Wan, X.; Yang, H.; Wang, Z. Dietary lycopene supplementation could alleviate aflatoxin b1 induced intestinal damage through improving immune function and anti-oxidant capacity in broilers. Animals 2021, 11, 3165. [CrossRef] [PubMed]

23. Li, S.; Luo, X.; Liu, B.; Crenshaw, T.D.; Kuang, X.; Shao, G.; Yu, S. Use of chemical characteristics to predict the relative bioavailability of supplemental organic manganese sources for broilers. J. Anim. Sci. 2004, 82, 2352. [CrossRef] [PubMed]

24. Olgun, O. Manganese in poultry nutrition and its effect on performance and eggshell quality. Worlds Poult. Sci. J. 2017, 73, 45-56. [CrossRef]

25. Lu, L.; Ji, C.; Luo, X.; Liu, B.; Yu, S. The effect of supplemental manganese in broiler diets on abdominal fat deposition and meat quality. Anim. Feed Sci. 2006, 129, 49-59. [CrossRef]

26. Berta, E.; Andrásofszky, E.; Bersényi, A.; Glavits, R.; Gaspardy, A.; Fekete, S.G. Effect of inorganic and organic manganese supplementation on the performance and tissue manganese content of broiler chicks. Acta Vet. Hung. 2004, 52, 199-209. [CrossRef]

27. Pacheco, B.; Nakagi, V.; Kobashigawa, E.; Caniatto, A.; Faria, D.; Faria Filho, D. Dietary levels of zinc and manganese on the performance of broilers between 1 to 42 days of age. Braz. J. Poult. Sci. 2017, 19, 171-178. [CrossRef]

28. Meng, T.; Gao, L.; Xie, C.; Xiang, Y.; Huang, Y.; Zhang, Y.; Wu, X. Manganese methionine hydroxy analog chelated affects growth performance, trace element deposition and expression of related transporters of broilers. Anim. Nutr. 2021, 7, 481-487. [CrossRef]

29. Ognik, K.; Kozłowski, K.; Stępniowska, A.; Szlązak, R.; Tutaj, K.; Zduńczyk, Z.; Jankowski, J. The effect of manganese nanoparticles on performance, redox reactions and epigenetic changes in turkey tissues. Animal 2019, 13, 1137-1144. [CrossRef]

30. Conly, A.; Poureslami, R.; Koutsos, E.; Batal, A.; Jung, B.; Beckstead, R.; Peterson, D. Tolerance and efficacy of tribasic manganese chloride in growing broiler chickens. Poult. Sci. 2012, 91, 1633-1640. [CrossRef] [PubMed]

31. Jankowski, J.; Ognik, K.; Stępniowska, A.; Zduńczyk, Z.; Kozłowski, K. The effect of the source and dose of manganese on the performance, digestibility and distribution of selected minerals, redox, and immune status of turkeys. Poult. Sci. 2019, 98, 1379-1389. [CrossRef]

32. Lu, L.; Luo, X.; Ji, C.; Liu, B.; Yu, S. Effect of manganese supplementation and source on carcass traits, meat quality, and lipid oxidation in broilers. J. Anim. Sci. 2007, 85, 812-822. [CrossRef] [PubMed]

33. Ghosh, A.; Mandal, G.; Roy, A.; Patra, A. Effects of supplementation of manganese with or without phytase on growth performance, carcass traits, muscle and tibia composition, and immunity in broiler chickens. Livest. Sci. 2016, 191, 80-85. [CrossRef]

34. Matuszewski, A.; Łukasiewicz, M.; Łozicki, A.; Niemiec, J.; Zielińska-Górska, M.; Scott, A.; Chwalibog, A.; Sawosz, E. The effect of manganese oxide nanoparticles on chicken growth and manganese content in excreta. Anim. Feed Sci. Technol. 2020, $268,114597$. [CrossRef]

35. Li, S.; Lin, L.; Liao, X.; Gao, T.; Wang, F.; Zhang, L.; Lin, X.; Liu, S.; Luo, X. Manganese elevates manganese superoxide dismutase protein level through protein kinase $C$ and protein tyrosine kinase. Biometals 2016, 29, 265-274. [CrossRef] [PubMed] 
36. Qin, S.Z.; Liao, X.D.; Lin, L.; Zhang, L.Y.; Lin, X.; Guo, Y.L.; Luo, X.G. Manganese enhances the expression of the manganese superoxide dismutase in cultured primary chick embryonic myocardial cells. J. Integr. Agric. 2017, 16, 2038-2046. [CrossRef]

37. Bozkurt, Z.; Bulbul, T.; Bozkurt, M.F.; Bulbul, A.; Maralcan, G.; Qeukeloglu, K. Effects of organic and inorganic manganese supplementation on bone characteristics, immune response to vaccine and oxidative stress status in broiler reared under high stocking density. Kafkas Univ. Vet. Fak. Derg. 2015, 21, 623-630.

38. Li, S.F.; Lu, L.; Hao, S.; Wang, Y.; Zhang, L.; Liu, S.; Liu, B.; Li, K.; Luo, X. Dietary manganese modulates expression of the manganese-containing superoxide dismutase gene in chickens. J. Nutr. 2011, 141, 189-194. [CrossRef] [PubMed]

39. Jiang, W.D.; Wu, P.; Tang, R.J.; Liu, Y.; Kuang, S.Y.; Jiang, J.; Tang, L.; Tang, W.N.; Zhang, Y.A.; Zhou, X.Q. Nutritive values, flavor amino acids, healthcare fatty acids and flesh quality improved by manganese referring to up-regulating the antioxidant capacity and signaling molecules TOR and Nrf2 in the muscle of fish. Food Res. Int. 2016, 89, 670-678. [CrossRef]

40. Liu, R.; Jin, C.; Wang, Z.; Wang, Z.; Wang, J.; Wang, L. Effects of manganese deficiency on the microstructure of proximal tibia and OPG/RANKL gene expression in chicks. Vet. Res. Commun. 2015, 39, 31-37. [CrossRef] [PubMed]

41. Wang, J.; Wang, Z.Y.; Wang, Z.J.; Liu, R.; Liu, S.Q.; Wang, L. Effects of manganese deficiency on chondrocyte development in tibia growth plate of Arbor Acres chicks. J. Bone Miner. Metab. 2015, 33, 23-29. [CrossRef]

42. Zhaojun, W.; Lin, W.; Zhenyong, W.; Jian, W.; Ran, L. Effects of manganese deficiency on serum hormones and biochemical markers of bone metabolism in chicks. J. Bone Miner. Metab. 2013, 31, 285-292. [CrossRef] [PubMed]

43. Lotfi, L.; Zaghari, M.; Zeinoddini, S.; Shivazad, M.; Davoodi, D. Comparison dietary nano and micro manganese on broilers performance. In Proceedings of the 5th International Conference on Nanotechnology: Fundamentals and Applications, Prague, Czech Republic, 11-13 August 2014; p. 293.

44. Wang, Y.; Gou, Z.; Lin, X.; Fan, Q.; Ye, J.; Jiang, S. Optimal Level of Supplemental Manganese for Yellow-Feathered Broilers during the Growth Phase. Animals 2021, 11, 1389. [CrossRef] [PubMed]

45. Mirzavandi Chegeni, M.; Mottaghitalab, M.; Hosseini Moghaddam, S.H.; Golshekan, M. Broiler intestine DMT1 gene expression and bone characteristics, as affected by in ovo injection of different forms of manganese. Ital. J. Anim. Sci. 2019, 18, 1215-1222. [CrossRef]

46. Sunder, G.S.; Panda, A.K.; Gopinath, N.C.S.; Raju, M.V.L.N.; Rao, S.V.R.; Kumar, C.V. Effect of supplemental manganese on mineral uptake by tissues and immune response in broiler chickens. J. Poult. Sci. 2006, 43, 371-377. [CrossRef]

47. Lu, L.; Wang, R.L.; Zhang, Z.J.; Steward, F.A.; Luo, X.G.; Liu, B. Effect of dietary supplementation with copper sulfate or tribasic copper chloride on the growth performance, liver copper concentrations of broilers fed in floor pens, and stabilities of vitamin $\mathrm{E}$ and phytase in feeds. Biol. Trace Elem. Res. 2010, 138, 181-189. [CrossRef] 